\title{
MORPHOLOGY AND PHYSICS OF SUPERNOVA REMNANTS
}

\author{
B. ASCHENBACH \\ Max-Planck-Institut für extraterrestrische Physik \\ 8046 Garching, Germany
}

\begin{abstract}
Spectrally resolved X-ray images of the Cygnus Loop and the Vela supernova remnant have been obtained during the ROSAT all sky survey. The remnants show highly filamentary brightness distributions. Temperatures between $1 \cdot 10^{6} \mathrm{~K}$ and $10 \cdot 10^{6} \mathrm{~K}$ have been measured. Both remnants show significant deviation from thermal pressure equilibrium. The highest pressure excursions of up to a factor of 50 are associated with the X-ray brightest filaments. Pressure variations are expected for very recently shocked clouds, on a smaller scale though. In a limited search of the all sky survey data 14 extended objects have been found, which have tentatively been identified as previously unknown supernova remnants. A total number of $\sim 60$ new remnants are expected from the analysis of the full sky.
\end{abstract}

\section{Introduction}

Supernova remnants (SNRs) have been known as high luminosity X-ray sources since the discovery of the X-ray emission from the Crab Nebula in 1963. In fact, together with the $\mathrm{X}$-ray binaries SNRs constitute the class of the most luminous objects in the galaxy, the Magellanic clouds and M31. The majority of the SNRs emit thermal radiation from a hot optically thin plasma with temperatures in the range of $\sim 1-10 \cdot 10^{6} \mathrm{~K}$. The emission therefore peaks at energies between $\sim 0.1$ and a few $\mathrm{keV}$. The remainder of the remnants show synchrotron emission with a power law energy spectrum decreasing with energy so that the bulk of the emission is found at the lowest energies. However, the amount of interstellar absorption effectively limits the low energy flux.

For an interstellar hydrogen column density $\leq 10^{22} \mathrm{~cm}^{-2}$ the band pass $(0.1-2.4 \mathrm{keV})$ of the ROSAT X-Ray Telescope (XRT) is quite suitable for an efficient detection of SNRs. Furthermore, the fast optics of the telescope (Aschenbach, 1988), the large field of view, the very low non-X-ray detector background (Aschenbach, 1991) and the imaging capabilities of the XRT are of greatest benefit for the detection and detailed study of SNRs, even for those of very low surface brightness. With the position sensitive proportional counter (PSPC), Pfeffermann et al. (1986), spectrally resolved images of a SNR can be taken to determine temperature, density and pressure maps with a spatial resolution down to 30 arcsec. With the high resolution imager (HRI) the morphology of SNRs can be studied down to the resolution limit of $\sim 5$ arcsec. In the scan mode of the telescope during the all sky survey the large diameter remnants like the North Polar Spur $\left(\mathrm{d} \sim 100^{\circ}\right)$, the Monogem Ring (d $\left.\sim 20^{\circ}\right)$, the Cygnus Super-Bubble $\left(\mathrm{d} \sim 13^{\circ}\right)$, the Cygnus Loop $\left(\mathrm{d} \sim 3^{\circ} \times 4^{\circ}\right)$, the Vela 
SNR ( $d \sim 7.3^{\circ}$ ), e.g., have been imaged completely for the first time, such that their full extent and the associated structure is now evident.

Clearly, the ROSAT results will advance our understanding of the scientific topics associated with SNRs such as stellar evolution, nucleogenesis, acceleration and propagation of cosmic rays, energetics and structure of the interstellar medium and star formation. In the following a few highlights will be presented from the analysis of the Cygnus Loop and the Vela SNR, and a preliminary account will be given of the statistics of galactic SNRs derived from the all sky survey data.

\section{The Cygnus Loop}

The Cygnus Loop is generally called a middle-aged SNR of $\sim 10,000$ to 20,000 years age at a distance of $\sim 770 \mathrm{pc}$. X-ray emission from the loop has been discovered already in 1970 (Grader et $a l$.); the first X-ray image of a few arcmin spatial resolution has been obtained with the Einstein IPC by Ku et al. (1984). During the ROSAT all sky survey the Cygnus constellation was scanned in November 1990 and a PSPC image of the Cygnus Loop was obtained as shown in Fig. 1. The effective spatial resolution is $\sim 1$ arcmin half power radius, uniform across the image.

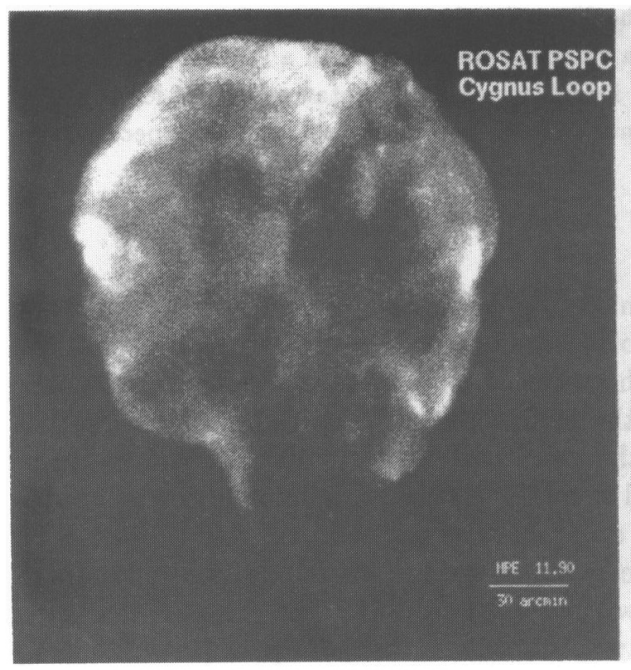

Figure 1. X-ray image of the Cygnus Loop.

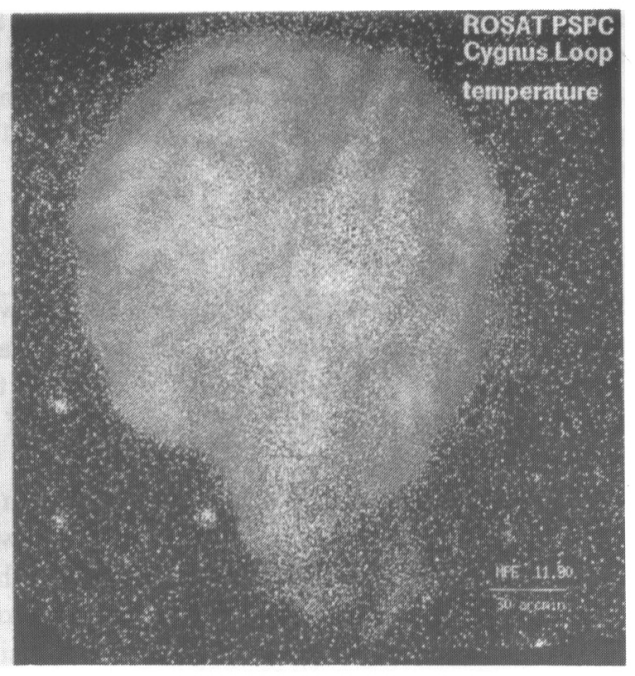

Figure 2. Temperature distribution in the Cygnus Loop. Darkest grey corresponds to $1 \cdot 10^{6} \mathrm{~K}$, white to $10 \cdot 10^{6} \mathrm{~K}$.

The surface brightness is highest in the north-east, which corresponds to NGC 6992. The brightness is lowest in the center part and it varies along the rim. The $\mathrm{X}$-ray bright regions tend to resolve into filamentary structure, such that the $\mathrm{X}$-ray image is very reminiscent of the optical $\mathrm{H}_{\alpha}$ image. However, there are also optical filaments, which have no X-ray counterpart and vice versa. The X-ray prominent V-type structure close to the south-west rim is not visible in the optical image. 
The claim that the X-ray emitting plasma in the Cygnus Loop is not isothermal has first been made by Kayat et al. (1980) using data from a sounding rocket flight. Temperature variations were confirmed by Charles et al. (1985) using the Einstein IPC data. Leahy et al. (1990) analysed non-imaging data from the HEAO 1 A-2 experiment and find that a twotemperature equilibrium plasma of $\sim 2.3 \cdot 10^{6} \mathrm{~K}$ and $12.6 \cdot 10^{6} \mathrm{~K}$ with very small variations across the remnant fits the data best. However, they claim a significant variation of low energy absorption by interstellar matter across the Loop.

The ROSAT PSPC data have been used to determine the temperature of the X-ray emitting plasma in each individual image pixel. A measure for the temperature is the energy per X-ray count, the calibration of which has been computed from a one-temperature Raymond-Smith emission code assuming standard cosmic elemental abundances. Of course, the calibration changes with the amount of interstellar column density $\mathrm{N}_{\mathrm{H}}$. Fig. 2 shows the unsmoothed temperature distribution assuming a uniform $\mathrm{N}_{\mathrm{H}}$. The temperature ranges from $1-10 \cdot 10^{6} \mathrm{~K}$. The temperature is lowest around the rim and at the very bright spots on the eastern and western boundary. The hottest regions with $\sim 20$ arcmin diameter are located very close to the center. Generally speaking the interior of the remnant appears to be the hottest part, whereas the regions with bright filamentary structure are significantly cooler. Given this wide temperature spread it is obvious that spatially non-resolved energy spectra, like the HEAO 1 A-2 measurements (Leahy et al.), cannot be fitted with a onetemperature model, but by models of two temperatures at least. Although detailed spectral fitting to the ROSAT data has not yet been performed there is evidence that $\mathrm{N}_{H}$ variations across the remnant, which would change the calibration between temperature and energy per X-ray count, are not significant. The rim, which is most likely to be at the same temperature along the circumference, shows indeed a value for the energy per X-ray count which is constant all around the circumference.

Generally, there is a tendency that bright regions show low temperatures and vice versa. However, this anticorrelation is not strictly fulfilled as it would be expected from exact pressure equilibrium. A map of the thermal pressure associated with the X-ray emitting plasma has been computed from the brightness per pixel, which is proportional to the square of the matter density times the length of the line of sight through the emitting region, and the temperature determined from the energy per X-ray count. The resulting map, which is pressure times square root of the depth of the emission region, shows large variations of up to a factor of $\sim 50$. Some of the variations may be produced by variations in the depth of the emission region from one image pixel to the other, a major fraction is due to variations of the thermal pressure, though. The lowest pressure is found in the fainter parts of the remnant; the filaments are slightly higher in pressure and the highest pressure appears in general in the brightest parts of the filaments. Exceptions, however, exist as well. The pressure in the bright V-type structure close to the south-west rim is not constant, but it is significantly higher in the eastern wing of the V. Obviously, the Cygnus Loop as a whole is not in pressure equilibrium as far as the thermal pressure of the X-ray plasma is concerned.

Pressure variations are expected from the interaction of the supernova blast wave with interstellar clouds of densities higher than the ambient intercloud medium. McKee and Cowie (1975) calculate thermal pressure variations in a cloudy medium of up to a factor of 3.15 for freshly shocked clouds. Using this interpretation the high pressure clouds must have been shocked only very recently. A detailed analysis of the ROSAT observations is still to be done. In particular the amount of pressure variation and the dependence on 
density / temperature is still to be understood.

\section{The Vela Supernova Remnant}

The Vela supernova remnant is one of the rare examples of SNRs, which show a thermal, large diameter partial X-ray shell containing a pulsar and a small synchroton nebula probably powered by the pulsar (Harnden et al., 1985). An X-ray image of the Vela SNR has been obtained by a series of 36 mosaic pointings with the Einstein IPC (Kahn et al., 1985). The Vela constellation was scanned by ROSAT in November 1990 and the image obtained during the all sky survey is shown in Fig. 3. The image also contains the SNR Puppis-A at the north-west edge of Vela. The grey scale in the image has been chosen to display just the brighter parts, such that the filamentary structure of the emission shows up. Similiar to the Cygnus Loop quite a number of individual bright filaments are visible. In contrast to the Cygnus Loop the boundary of the Vela remnant is not that well defined, in particular the southern region, which apears rather irregular in shape. Of particular interest is the eastern rim, from which a wedge type structure protrudes almost by $1^{\circ}$ showing a bright patch on its outward boundary. A similar wedge type structure, though much fainter, is visible further up north offset from the rim by $\sim 1.5^{\circ}$.

Fig. 4 is a different display of the ROSAT Vela image which emphasizes the low brightness regions. From this figure the full extent of the Vela SNR becomes evident. The very faint western parts are enclosed by a long arc like filament running south from the PuppisA position. The existence of this arc has already been mentioned by Seward (1991), who associated the arc with the Vela SNR based on its soft Vela type IPC X-ray spectrum. An eye-ball fit circle has been included in Fig. 4, which outlines the boundary of the Vela SNR. The remnant fits nicely into this circle, which has a diameter of $7.3^{\circ}$. Obviously the remnant is significantly larger than $5.5^{\circ}$ previously derived from radio and optical images. Fig. 4 shows additional patchy emission outside of the circle, particularly in the west and southeast, and it is not clear whether this emission is associated with the Vela supernova event. Possibly this emission is a signal from the Gum nebula, seen for the first time in $\mathrm{X}$-rays. However, further analysis on a much larger sky area around Vela is required to demonstrate the existence of X-ray emitting plasma associated with the Gum nebula.

As described in the previous chapter on the Cygnus Loop temperature and pressure maps have been built for the Vela remnant. Temperature variations across the remnant are evident as well as pressure variations. However, the range of pressure variations is less for the Vela remnant and particularly high pressure is found only for small size regions within the bright filament area in the north and southeast of the remnant. As in the case of the Cygnus Loop further analysis is required to understand the results in the framework of the interaction of the blast wave with a cloudy medium.

Based on the fact that the Vela pulsar is offset from the center of the radio and optical image by $\sim 1.4^{\circ}$, Bignami and Caraveo (1988) have discussed the possibility that the Vela pulsar is not associated with the Vela remnant. However, the new X-ray boundary of the Vela remnant shows that the pulsar is located much closer to the center. In fact, the circle drawn in Fig. 4 is centered on the pulsar position. This position seems to be not optimal and the explosion center might have been somewhat further south, but the difference is less than 20 arcmin. This difference can be explained reasonably well by a proper motion of the pulsar. Assuming an age of 15,000 years and a distance of $500 \mathrm{pc}$ the transverse velocity required to produce the 20 arcmin distance is computed to be $190 \mathrm{~km} \mathrm{~s}^{-1}$. This value is quite comparable with the value measured for the Crab pulsar which amounts to 
$(125 \pm 30) \mathrm{km} \mathrm{s}^{-1}$ (Trimble, 1971). Bignami and Caraveo have also measured optically an upper limit of the present proper motion of the Vela pulsar of $0.06 \operatorname{arcsec}_{\mathrm{yr}^{-1}}$ with respect to the adjacent star field. The upper limit of 20 arcmin for the offset and an age of 15,000 years corresponds to an upper limit of $0.08 \operatorname{arcsec} \mathrm{yr}^{-1}$. From this point of view the association of the pulsar with the remnant cannot be questioned. Because of the proximity of the pulsar position and the explosion center also no unrealistically high pulsar space velocity (Harnden et al., 1985) has to be invoked to maintain the association.

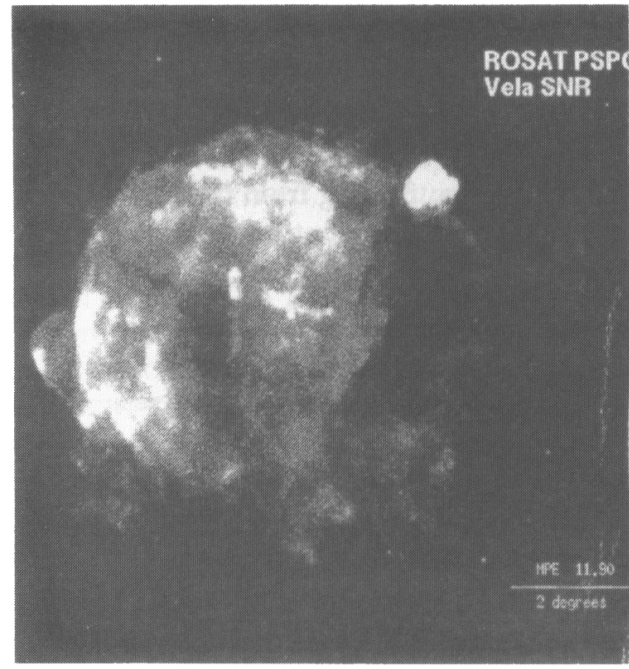

Figure 3. X-ray image of the Vela supernova remnant; Puppis-A shows up overexposed at the north-west edge.

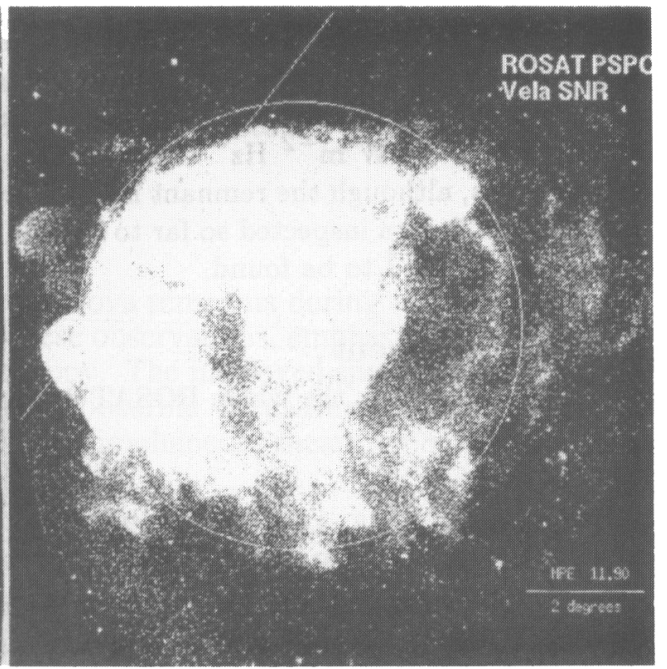

Figure 4. X-ray image of the Vela SNR, enhanced to show the faintest structures; circle is centered on Vela pulsar position; straight line marks the galactic plane.

\section{Statistics of Galactic Supernova Remnants}

The ROSA'T all sky survey offers for the first time to search the galaxy systematically for $\mathrm{X}$-ray emitting SNRs. Previously this has been done at radio frequencies and Green (1991) has compiled a list of galactic radio emitting SNRs. Out of the 175 radio sources listed, 47 remnants have been detected and imaged in X-rays before ROSAT (Seward, 1990).

So far, the ROSAT all sky survey data have been checked at 112 positions of the known radio remnants and $55 \mathrm{X}$-ray sources have been detected, out of which 27 sources were not known previously as $\mathrm{X}$-ray sources. The detection rate outside the galactic center region is significantly higher. Outside the region defined by $\left[|\mathrm{l}| \leq 25^{\circ},|\mathrm{b}| \leq 5^{\circ}\right] 67$ radio positions have been inspected and $48 \mathrm{X}$-ray counterparts have been found, which equals a detection rate of $72 \%$. 22 of the $48 \mathrm{X}$-ray sources have been observed for the first time in X-rays. One example is $\mathrm{G} 166.0+4.3$, which shows a peculiar morphology at radio frequencies (Pineault et al., 1987). A very similiar structure is observed in the ROSAT 
$\mathrm{X}$-ray image of this object.

Inside the region $\left[|1| \leq 25^{\circ},|\mathrm{b}| \leq 5^{\circ}\right]$ only $7 \mathrm{X}$-ray sources have been found for a total of 45 radio remnant positions. This low detection rate is mostly likely due to high interstellar absorption implying that the vast majority of these radio remnants are at a distance $>4 \mathrm{kpc}$.

During the inspection of the ROSAT all sky survey data for X-ray counterparts of known radio SNRs 14 extended X-ray sources have been found serendipitously, which have no known radio counterparts. Because of their sizes these sources are most likely SNRs, although a spectral analysis is still to be done to discriminate them from clusters of galaxies. One of these SNRs discovered by the X-ray emission is G156.2+5.7 (Pfeffermann et al., 1991). Follow-up observations at radio frequencies with the 100-m Effelsberg telescope have confirmed the identification as a SNR with an extremely low surface brightness at $1 \mathrm{GHz}$ of only $5.8 \cdot 10^{-23} \mathrm{~W} \mathrm{~m}^{-2} \mathrm{~Hz}^{-1} \mathrm{sr}^{-1}$ (Reich et al., 1991), which is the lowest among all known SNRs, although the remnant is quite bright in X-rays. Scaling from the limited area of the ROSAT data inspected so far to the full sky $\sim 60$ new X-ray but uncatalogued radio SNRs are expected to be found.

\section{Acknowledgement}

I would like to thank the whole ROSAT team at MPE for help in the data analysis.

\section{References}

Aschenbach, B., 1988, Appl. Optics 27, 1404.

Aschenbach, B., 1991, Rev. Modern Astron., 4, 173.

Bignami, G.F. and Caraveo., P.A., 1988, Astrophys. J. (Letters), 325, 5.

Charles, P., Kahn, S., and McKee, C., 1985, Astrophys. J., 295, 456.

Grader, R., Hill, R., and Stoering, J., 1970, Astrophys. J. (Letters), 161, 45.

Green, D.A., 1991, Publ. Astron. Soc. Pac., 103, 209.

Harnden, F.R., Jr., Grant, P.D., Kahn, S.M., and Seward, F.D., 1985, Astrophys. J., 299, 828.

Kahn, S.M., Gorenstein, P., Harnden, F.R., Jr., and Seward, F.D., 1985, Astrophys. J., 299, 821.

Kayat, M., Rolf, D., Smith, G., and Willingale, R., 1980,

Mon. Not. R. Astr. Soc., 191, 729.

Ku, W., Kahn, S., Pisarski, R., and Long, K., 1984, Astrophys. J., 278, 615.

Leahy, D.A., Fink, R., and Nousek, J., 1990, Astrophys. J., 363, 547.

McKee, C.F., and Cowie, L.L., 1975, Astrophys. J., 195, 715.

Pfeffermann, E., et al., 1986, Proc. SPIE 733, 519.

Pfeffermann, E., Aschenbach, B., and Predehl, P., 1991, Astron. Astrophys. (Letters), 246, 28.

Pineault, S., Landecker, T.L., and Routledge, D., 1987, Astrophys. J., 315, 580.

Reich, W., Fürst, E., and Arnal, E.M., 1991, Astron. Astrophys., , in press.

Seward, F.D., 1990, in Imaging X-Ray Astronomy, Elvis, M. (ed.),

Cambridge University Press, p241.

Seward, F.D., 1990, Astrophys. J. Suppl., 73, 781.

Trimble, V., 1971, in IAU Symposium No. 46, The Crab Nebula, Davies, R.D., and Smith, F.G. (eds.), D. Reidel Publ. Company, p12. 